\title{
N92-22435
}

\section{PHASED ARRAY ANTENNA BEAMFORMING USING OPTICAL PROCESSOR}

\author{
L. P. Anderson, F. Boldissar, D. C. D. Chang \\ Hughes Aircraft Company, Space and Communications Group \\ P. O. Box 92919, El Segundo, CA 90245
}

\begin{abstract}
This work concerns itself with the analytical investigation into the feasibility of optical processor based beamforming for microwave array antennas. The primary focus is on systems utilizing the $20 / 30 \mathrm{GHz}$ communications band and a transmit configuration exclusively to serve this band.

A mathematical model is developed for computation of candidate design configurations. The model is capable of determination of the necessary design parameters required for spatial aspects of the microwave "footprint" (beam) formation. Computed example beams transmitted from geosynchronous orbit are presented to demonstrate network capabilities. The effect of the processor on the output microwave signal to noise quality at the antenna interface is also considered.
\end{abstract}

\section{INTRODUCTION}

Phased array antennas are playing an increasingly important role in current and anticipated radar and communications applications. RF distribution manifolds used to furnish the required aperture excitation can become prohibitively bulky for space applications. There are also limitations on microwave bandwidth and speed of beam scanning/reconfigurability with conventional methods.

Problems with conventional beamforming for large arrays makes it imperative to develop a proposed technology that performs array beamforming via alternative approaches. A novel method utilizing an optical signal processor to furnish these excitations has been proposed.

Keopf [1] has described the basic concept for both transmit and receive applications. In his description, a heterodyne processing scheme is used in conjunction with a static means (i.e. pinhole mask) for production of a scaled image of the desired far field pattern.

The scope of this effort was to derive a transmit architecture which would be tractable to formation of generalized shaped beam far field patterns. Thus a dynamic means for image formation must be employed. Additionally, the system should be capable of operation in the $20 / 30 \mathrm{GHz}$ communications band. These requirements necessitated consideration of alternative approaches due to inherent limitations of the Keopf method in addressing these requirements. Once this basic processor configuration was established, a computer model of the network was generated for analysis of its beamforming capabilities.

A conceptual transmit mode processor is depicted in Figure 1. A continuous wave laser source emits a beam that is split into two paths. The optical signal in Path A undergoes temporal modulation with a microwave information signal. The optical signal in Path $\mathrm{B}$ is spatially modulated by a reflectance function, then Fourier transformed with a lens. The modulated optical signals from both of these paths are then added together and sampled spatially by a fiber optic array which transports the combined optical signal to the individual microwave array elements. At the array element the optical signal is detected to reproduce the microwave information signal. The spatial amplitude and phase distribution provided by Path B will be retained. This microwave signal is then amplified and radiated by the array antenna. 


\section{SPATIAL PROCESSING MODEL}

The model (Figure 2) for the optical processor is based on the Fourier transform relationship between the front and rear focal planes of the optical lens of focal length $f$ [2]. Here a two dimensional image formed in the rear focal plane can be expressible as an electric field distribution in the forward focal plane. The input field distribution is expressed as:

$$
E\left(x_{1}, y_{1}\right)=t_{0}\left(x_{1}, y_{1}\right) E_{0}
$$

where $t_{0}\left(x_{1}, y_{1}\right) \equiv$ image transmittance function of the SLM

the field formed in the front focal plane of the lens via diffraction are thus expressible through a Fourier transform i.c.,

$$
E\left(x_{2}, y_{2}\right) \sim \iint_{-\infty}^{+\infty} t_{0}\left(x_{1}, y_{1}\right) \exp \left\{-\frac{i z^{\pi}}{\lambda f}\left(x_{1} x_{2}+y_{1} y_{2}\right)\right\} d x_{1} d y_{l} \text { (2) }
$$

The pupil function is assumed to be unity for the lens. A digitized image of the desired far field footprint is provided in the form of light and dark contrasting elements (pixels). A discreet Fourier transform is thus formed for a NxM matrix scene of area axa (square) constant transmittance pixels. Thus the front focal field are now

$$
E_{\text {ton }}\left(x_{2}, y_{2}\right) \sim \sum_{n=1}^{N} \sum_{m=1}^{M} t_{n m}\left(x_{1}, y_{1}\right) \operatorname{sinc}\left(a f_{x_{n}}\right) \operatorname{sinc}\left(a f_{y m}\right) e^{j 2 \pi\left(b_{n} f_{x}+e_{n} f_{y}\right)}
$$

where $f_{x}, f_{y}$ are spatial frequencies and bm, cn are offsets of the $m$, $n$th pixel in the $x, y$, directions respectively. The function

$$
\sin C(z) \equiv \frac{\sin (\pi z)}{\pi z}
$$

Thus both amplitude and phase (i.e. pixel "brightness" and location relative to a central axis) is contained in equation (3). The distribution $E\left(x 2, y_{2}\right)$ now represents the complex aperture excitation, once downconverted, of the array antenna. The far zone fields resulting from such excitations may be obtained via standard Fraunhoffer diffraction evaluation of the array aperture.

Deterministic errors (i.e. axial defocussing of F.T.L.) can be approximated by the addition of a quadratic phase term to the incident wavefront i.e. [3]

$$
E_{n m}\left(x_{1}, y_{1}\right)=E_{0}\left(t_{n m}\left(x_{1}, y_{1}\right) e^{-j \delta_{n m}}\right) \quad(4)
$$

Here the quadratic phase term is defined as:

$$
\delta_{n m}\left(x_{1}, y_{1}\right)=k\left\{\frac{x_{1 n}^{2}+y_{1 m}^{2}}{2 R_{0}}\right\}
$$

where $\mathrm{k}$ is the free space wavenumber and $\mathrm{Ro}$ is the axial displacement of pixel from image plane. The effect of the error was evaluated using a one dimensional model to simplify understanding of the resultant Fourier transform spectra. For progressive values of phase error, the presence of grating lobes of increasing intensity is observed in the spatial frequency output plane. The element pattern presented by the individual pixels in the SLM serve to modulate the amplitude of the grating lobe formation. The existence of these lobes in the complex aperture distribution of the antenna array will serve to create secondary beams in the far field. Albeit in most applications, these lobes will not exist in visible space due to finite array aperture; they will tend to decrease main lobe directivity. 


\section{TEMPORAL PROCESSING MODEL}

An essential element of consideration in the utilization of the processor is the evaluation of signal quality in the communications link. The underlying basis of the analysis is the development of the expression for processor output signal to noise ratio. It can be shown [4] that exclusive of input and output amplifiers and their associated matching circuits, the expression for processor $(S / N)$ out is found to be

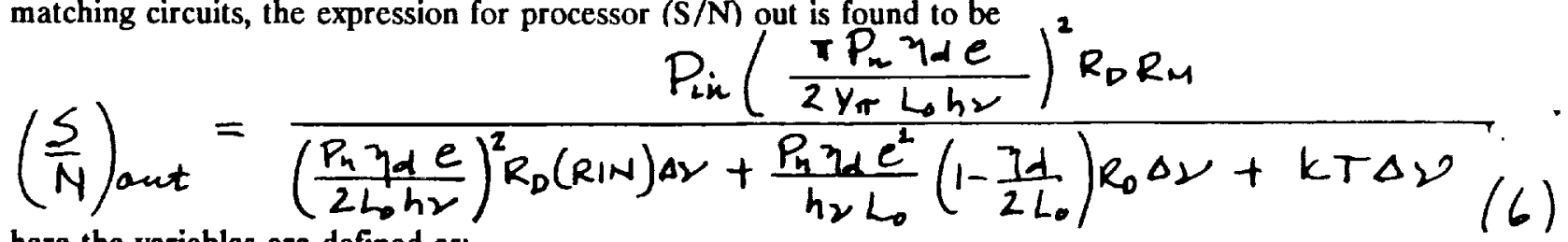

here the variables are defined as:

$$
\begin{aligned}
& P_{\text {in }} \quad=\quad \text { input RF signal drive power } \\
& \mathbf{P}_{\mathbf{n}} \quad=\quad \text { laser optical power at } n \text {th array element } \\
& \mathrm{N}_{\mathrm{c}} \quad=\quad \text { total number of microwave antenna array elements } \\
& \mathbf{R}_{\mathrm{D},} \mathbf{R}_{\mathrm{M}}=\text { resistance of photodiode and modulator respectively } \\
& V_{p}=\quad \text { voltage required for } 18000 \text { phase shift in } \mathrm{E} / \mathrm{O} \text { modulator } \\
& \Delta \mathrm{n} \quad=\quad \text { operating } \mathbf{R F} \text { bandwidth } \\
& \text { n }=\text { operating optical frequency } \\
& \mathrm{h}_{\mathrm{o}} \quad=\quad \text { photodiode efficiency } \\
& \mathbf{L}_{\mathbf{o}} \quad=\quad \text { total OPBFN optical losses } \\
& \text { RIN }=\text { laser random intensity noise } \\
& \mathrm{h}, \mathrm{K}=\text { Planck's and Boltzman constants (respectively) } \\
& \text { e }=\text { electronic charge }
\end{aligned}
$$

The noise contributions in the denominator can be attributed to three major catcgorics [5]; (1) Lascr noise, (2) partition noise (quantum effects) and (3) detector noise. Note that this general equation will be weighted accordingly with the individual amplitude coefficients associated with the F.T. spectra at the fiber optic input. Thus for uniform illumination:

$$
P_{T}=N_{e} P_{n}
$$

One can recognize the numerator as simply expressing the link gain i.e.;

$$
G_{\text {TOPBFN }}=\frac{P_{\text {RFAUT }}}{P_{\text {RFIN }}}=\left[\frac{\pi P_{m} \eta d e}{2 V_{\pi} L h_{2}}\right]^{2} R_{D} R_{M} \text { (8) }
$$

A key aspect of the indirect modulation scheme in the OPBFN is that kink gain can be controlled by input laser power. This condition allows OPBFN link gain of unity or greater for certain network configurations. This advantage is not realizable in direct modulation schemes for OPBFN's. Because of CW laser operation in the former, the system performance does not degrade at high frequencies. 
Utilization of equation (6) allows the study of $(\mathrm{S} / \mathrm{N})$ out as a function of input laser power. This can be expressed with the number of phases array elements as a parameter.

Figure 3 depicts this relationship. Here we have compared the effect of operational RF bandwidth on required optical power. We have observed that in general, the required optical power for a given array size and desired ( $\mathrm{S} / \mathrm{N}$ ) out is proportional to the square root of the bandwidth. Thus the smaller the desired bandwidth, the less optical power is needed. As can be seen, twice as much optical power is required to meet minimum (S/N) out when the bandwidth is raised from $250 \mathrm{MHz}$ to $1 \mathrm{GHz}$.

\section{RESULTS}

A theoretical model of a liquid crystal light valve (i.e. LCLV) was developed to study the aspects of beam formation using an optical processor based network. Figure 4 depicts a digitized image of a typical CONUS (Continental United States) footprint and selected regional coverages*. The chosen trial antenna aperture was a 121 element planar array, operating at a transmit frequency of $20 \mathrm{GHz}$. The total available aperture is 6273 square wavelengths. The particular grid density of $51 \times 23$ pixels in the LCLV array was chosen on the basis of the most efficient utilization of land mass coverage from the designated geosynchronous orbital position. Figures $5 \mathrm{a}, 5 \mathrm{~b}$, and $5 \mathrm{c}$ illustrate the versatility of the network by the creation of area coverage beams as well as multiple spot beams.

It might be noted that these simulations were done assuming an ideal LCLV with pixel transmittance being either 1 or 0 (i.e. infinite contrast ratio). In reality, there is always some leakage through the light valve so that the transmittance cannot go to zero. The effect of such leakage tends to broaden the main lobe and heighten the sidelobe levels of the microwave far field pattern. A parametric study was thus conducted to determine the lower limit of contrast ratio for adequate far field patterns to result. Criteria for evaluation were sidelobe level an main lobe beam width. It was determined that for the network under study, a minimum contrast ratio of $30 \mathrm{~dB}$ was required for spot beams and a $25 \mathrm{~dB}$ level for sector beams before a $10 \%$ increase in sidelobe level or half power beam width (as applicable) was observed.

A simple experiment was conducted to examine the validity of the theoretical spatial path model. Details of the complete evaluation can be found in [4]. A breadboard of the spatial path (i.e. Path B) was constructed on an optical bench and operated at $0.63 \mathrm{~m}$ using a standard He-Ne laser as the source illumination. An "off the shelf" Hughes model H01460 liquid crystal light valve was used to create images of full CONUS and quarter CONUS far field footprints. The output spatial frequency spectra (i.e. Fourier transform distribution) was used as the basis of comparison between theory and experiment. The model was adjusted to simulate a $20 \mathrm{~dB}$ background leakage in the LCLV image plane.

Initially photographs were taken to examine the qualitative nature of the optical transform spectra as compared to these computer predicted counterparts. The central lobe and first order sidelobe size and positions corresponded exactly with the predicted patterns once the photos were scaled. The scaling was necessary since the transform spectra were magnified through microscope objectives for evaluation of structural detail.

In order to check the quantitative nature of the computer predicted FT spectra at the processor output, measurement of electric field intensity of pattern cuts through both CONUS and quarter CONUS transforms were conducted. This was accomplished by using the digitizing CCD camera in conjunction with an oscilloscope for real time viewing of the voltage amplitudes. Horizontal pattern cuts were taken through both F.T. spectral outputs and exhibited reasonable agreement with computed intensities over the main lobe and first sidelobe regions. Figures 6 and 7 depict the comparison between computed and a measured output of the CCD camera for CONUS and quarter CONUS transform spectra respectively. As can be seen, dynamic range problems due to central lobe saturation of the camera prevented detailed pattern measurement beyond the second order lobe.

*Note that the image formed on the LCLV is an inverted scaled image of the desired far field pattern. 


\section{CONCLUSION}

The concept of optical processor based microwave antenna beamforming has been deemed feasible supported by the analysis presented herein. Fundamental concepts of the theoretical model have been validated via experiment. Advantages and disadvantages of the approach as referenced to more conventional designs have been highlighted to establish the validity of this technique.

A quantitative view of the processor characteristics at the output of the antenna was presented. As a result, a generalized computer model has been established to aid in the processor design procedure.

Refinement of the analytical model and test of a breadboard transmit processor comprises the bulk of future work to be done. This includes the determination of processor configurations for receive applications. The breadboard model shall and in the understanding of single sideband modulation requirements of the processor as well as path length sensitivity.

\section{REFERENCES}

1. Keopf. G. A., "Optical Processor for Phased Array Antenna Beam Formation", Proc. of SPIE Vol 477 - 1984

2. Goodman Introduction to Fourier Optics McGraw Hill - Cpy 1968

3. Casaseut, et al "Phased Error Model for Simple Fourier Transform Lenses" Applied Optics Vol 17, No, 11, June 1978

4. Anderson, et al. "Antenna Beamforming using Optical Techniques - Follow on Study" NASA - LeRc Contract \#NAS3-25720 Final Report - February 1991

5. Buckley, R. H. "Progress in Microwave Modulation Fiber Optic Links" Proc. SPIE Vol 1102, January 1979 


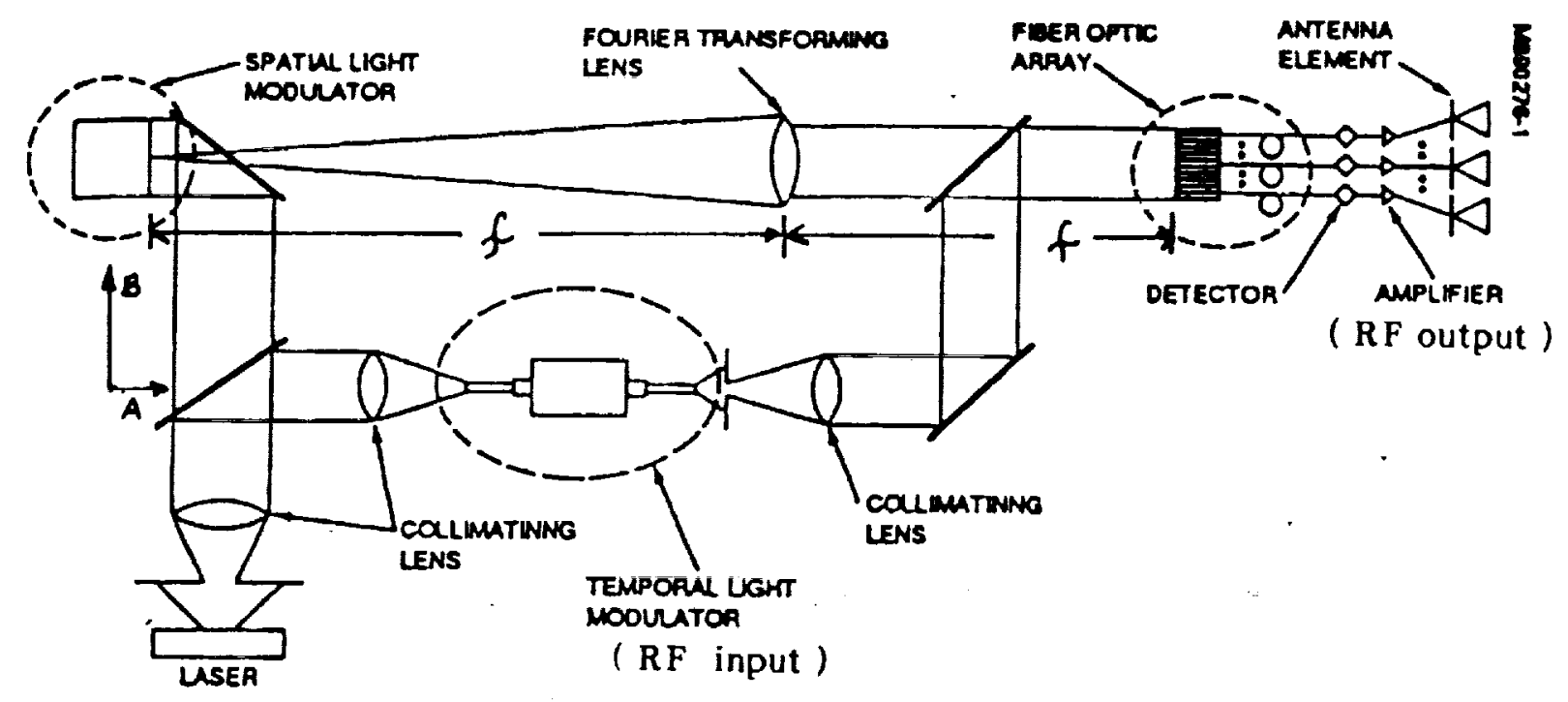

Figure 1 Optical Processor Beamfoming Network Schematic

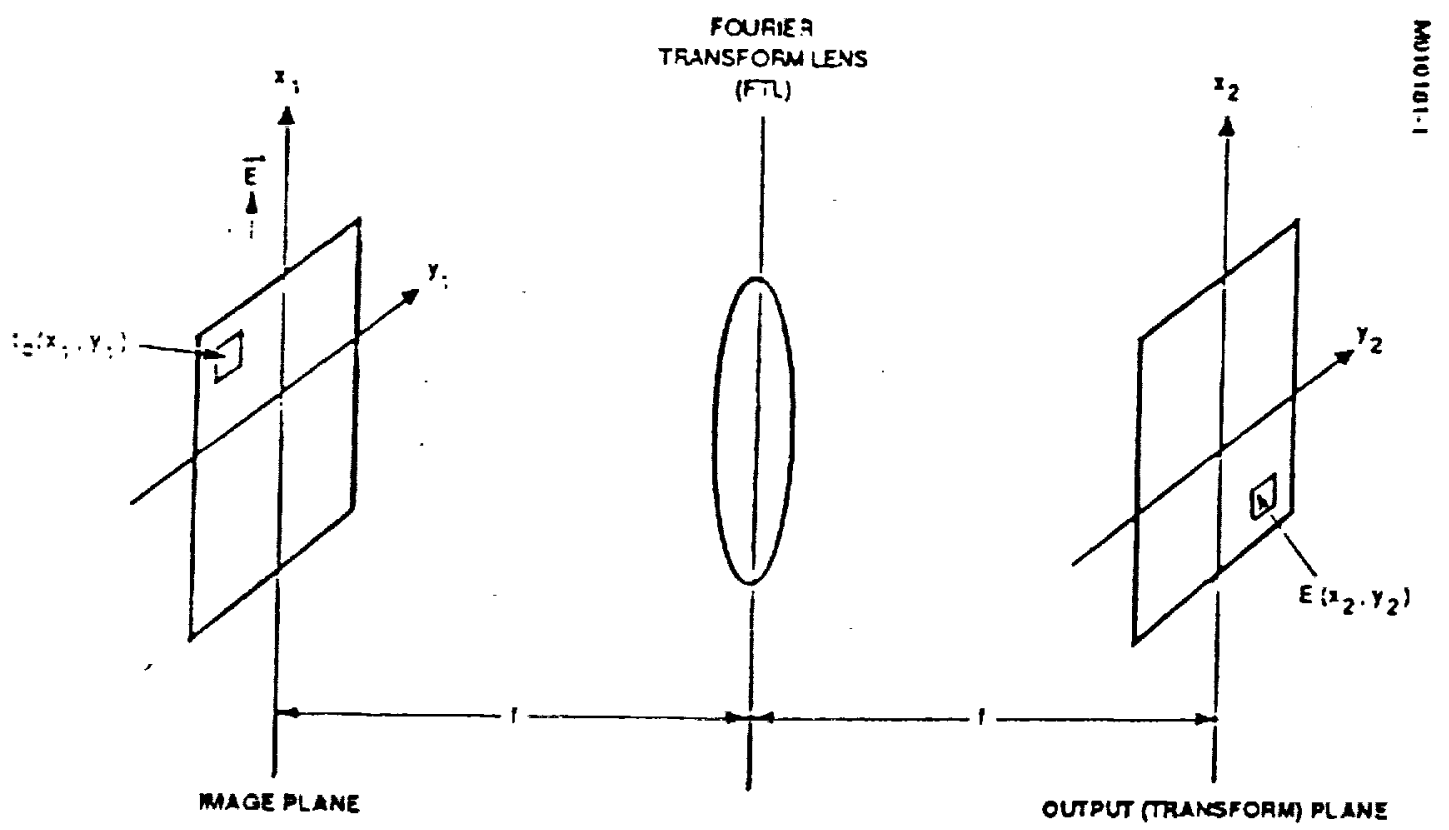

FTL FOCAL PLANE RELATIONSHIP

Figure 2 Spatial Processing Path Geometry 
For glven $\left(\frac{S}{N}\right)_{0}^{\dagger} ; P_{L} \propto \sqrt{\Delta v}$
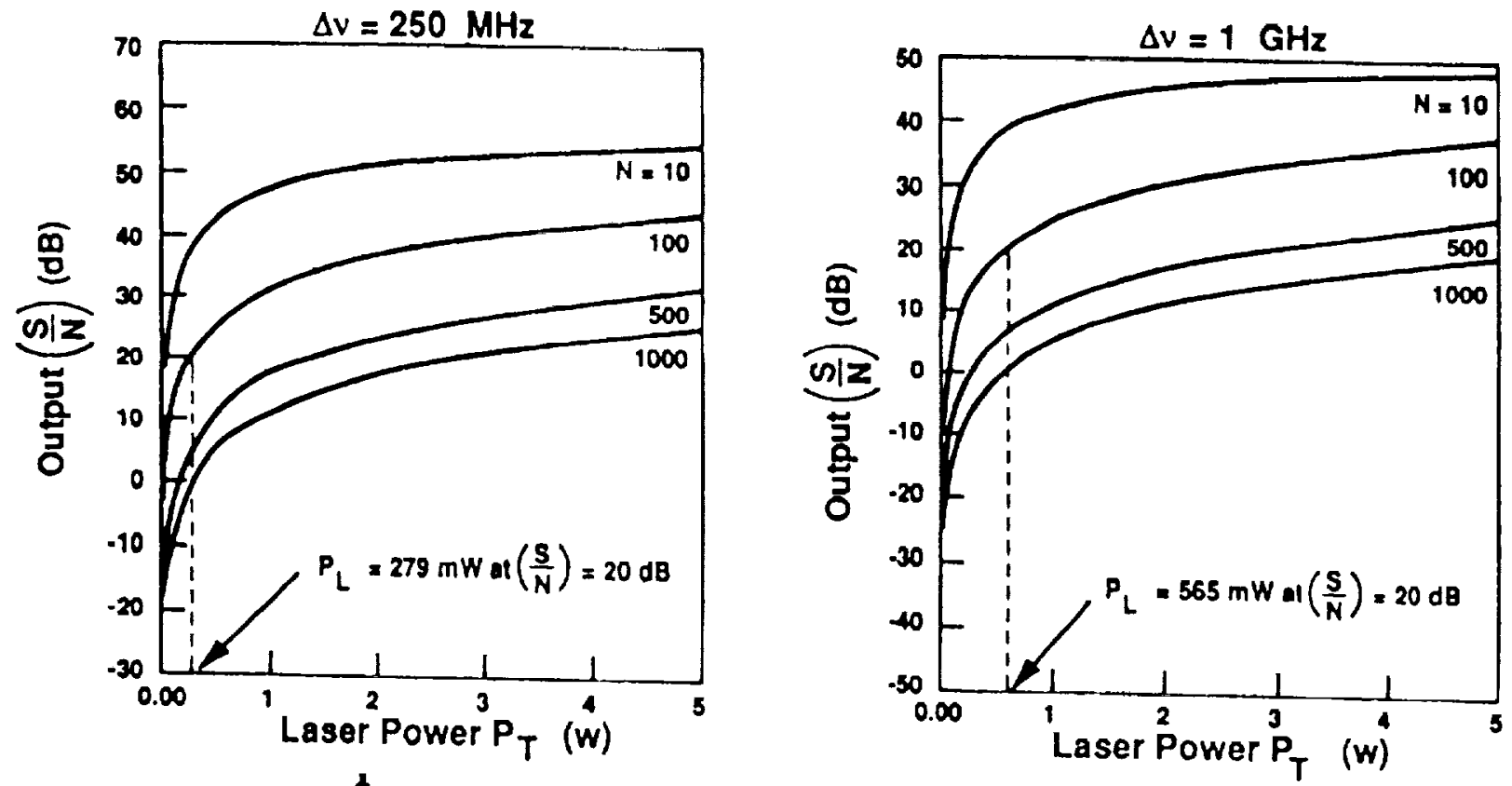

$1011043-02$

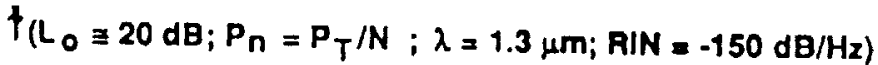

Figure 3 (S/N) Output vs. Required Laser Power

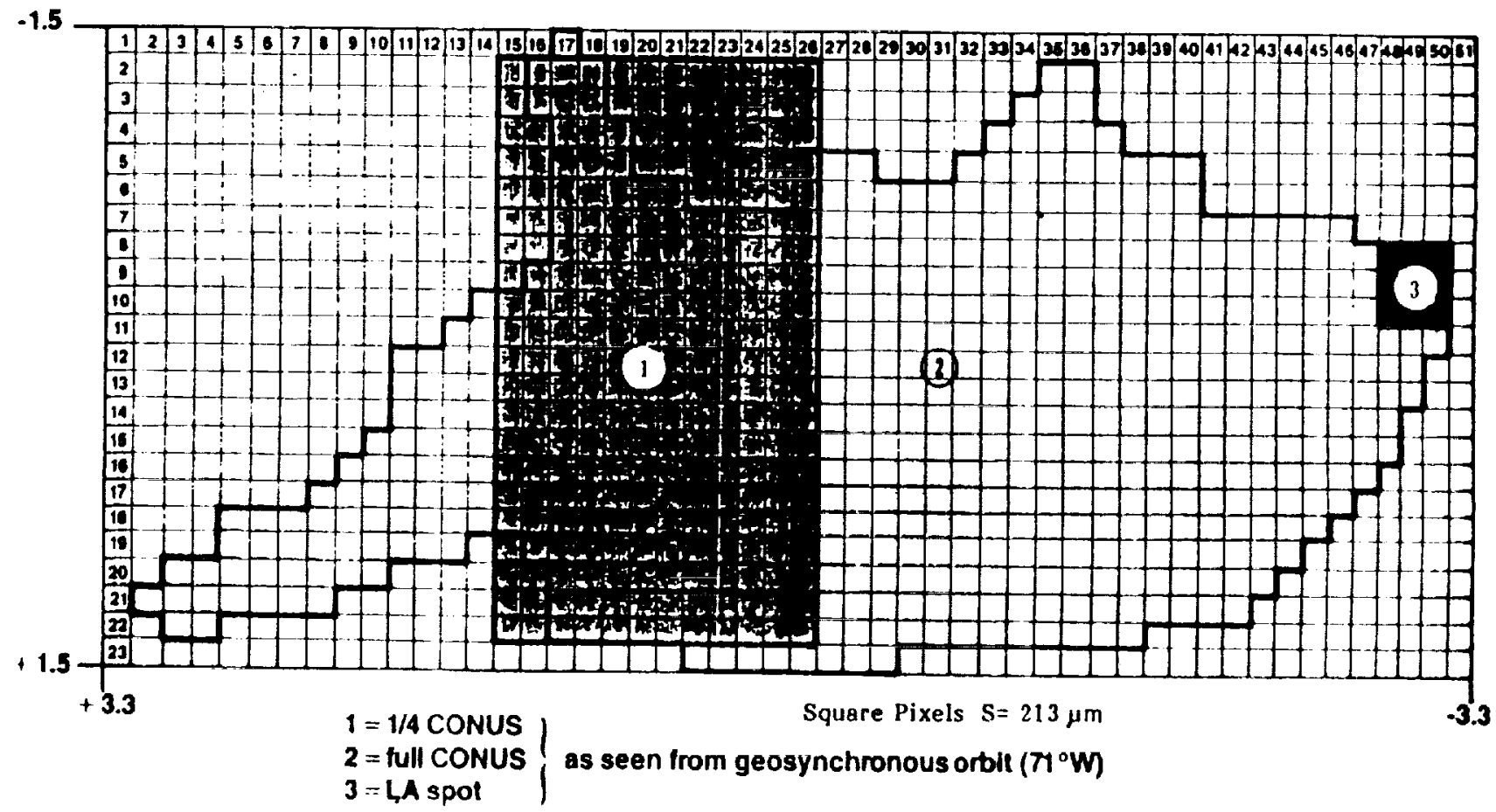

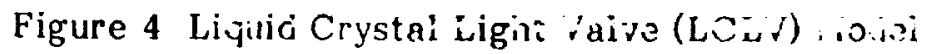




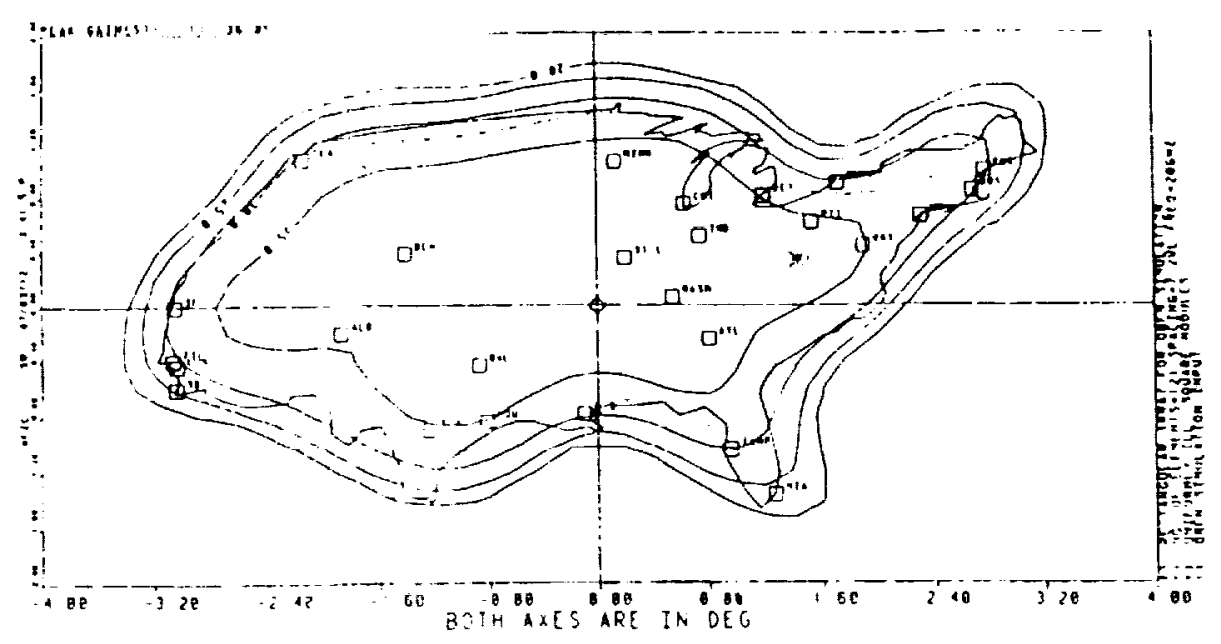

Figure 5a Full CONUS Beam Coverage

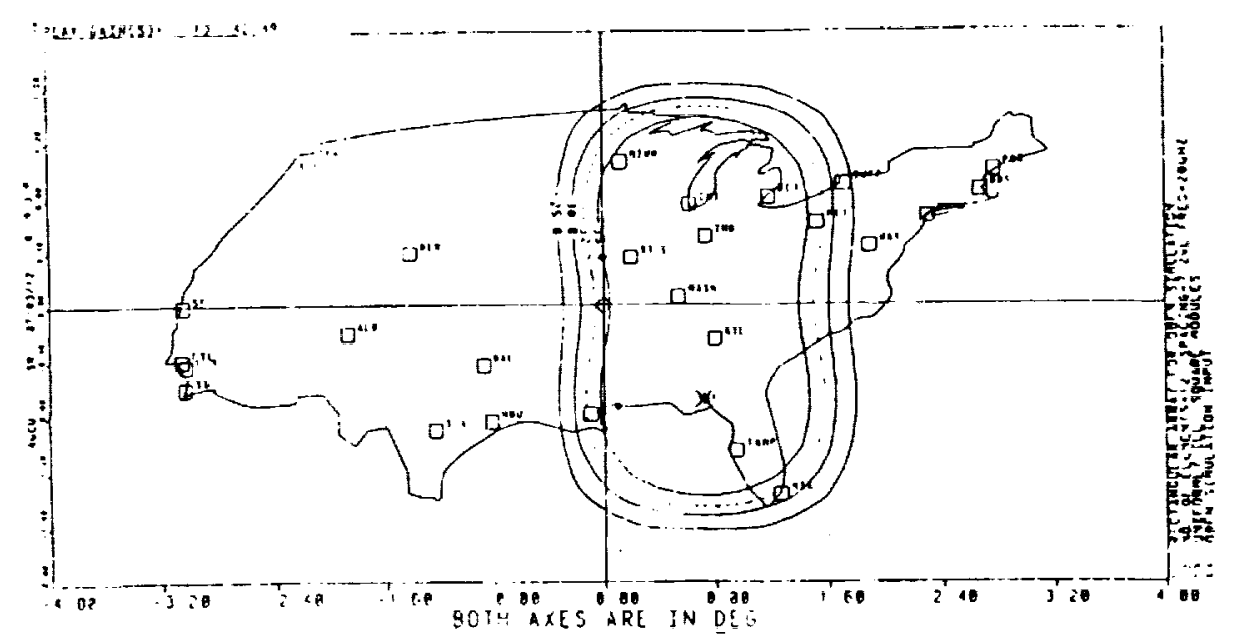

Figure 5b Midwestern Quarter CONUS Beam Coverage

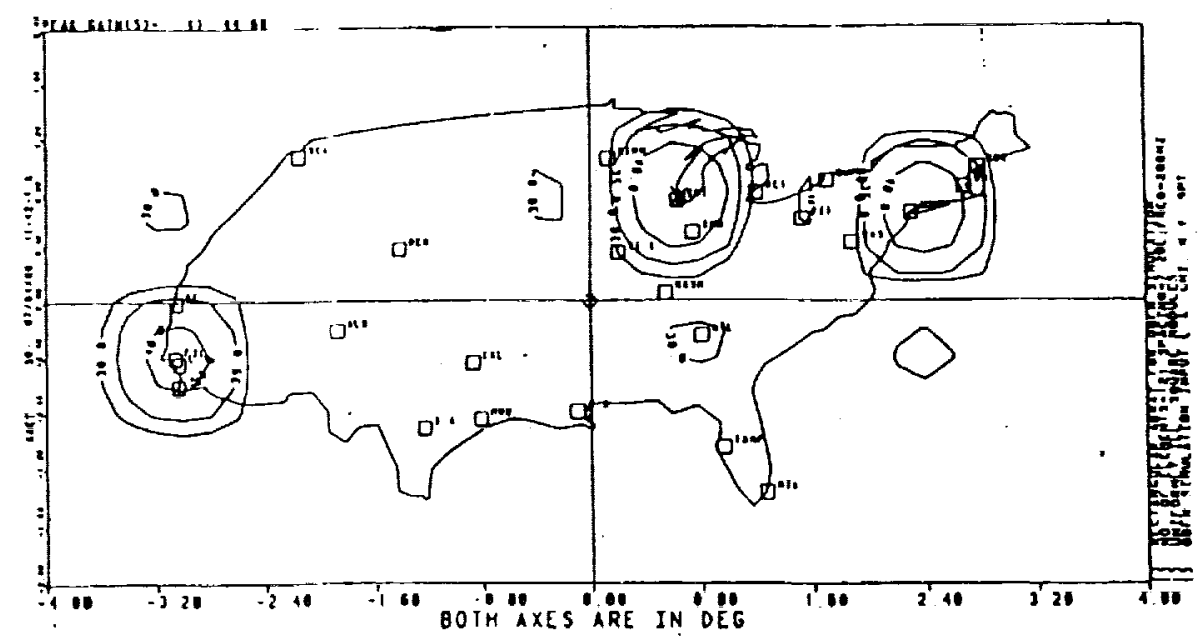

Figure 5c Multiple Spot Coverage (L.A./Chicago/N.Y.) 

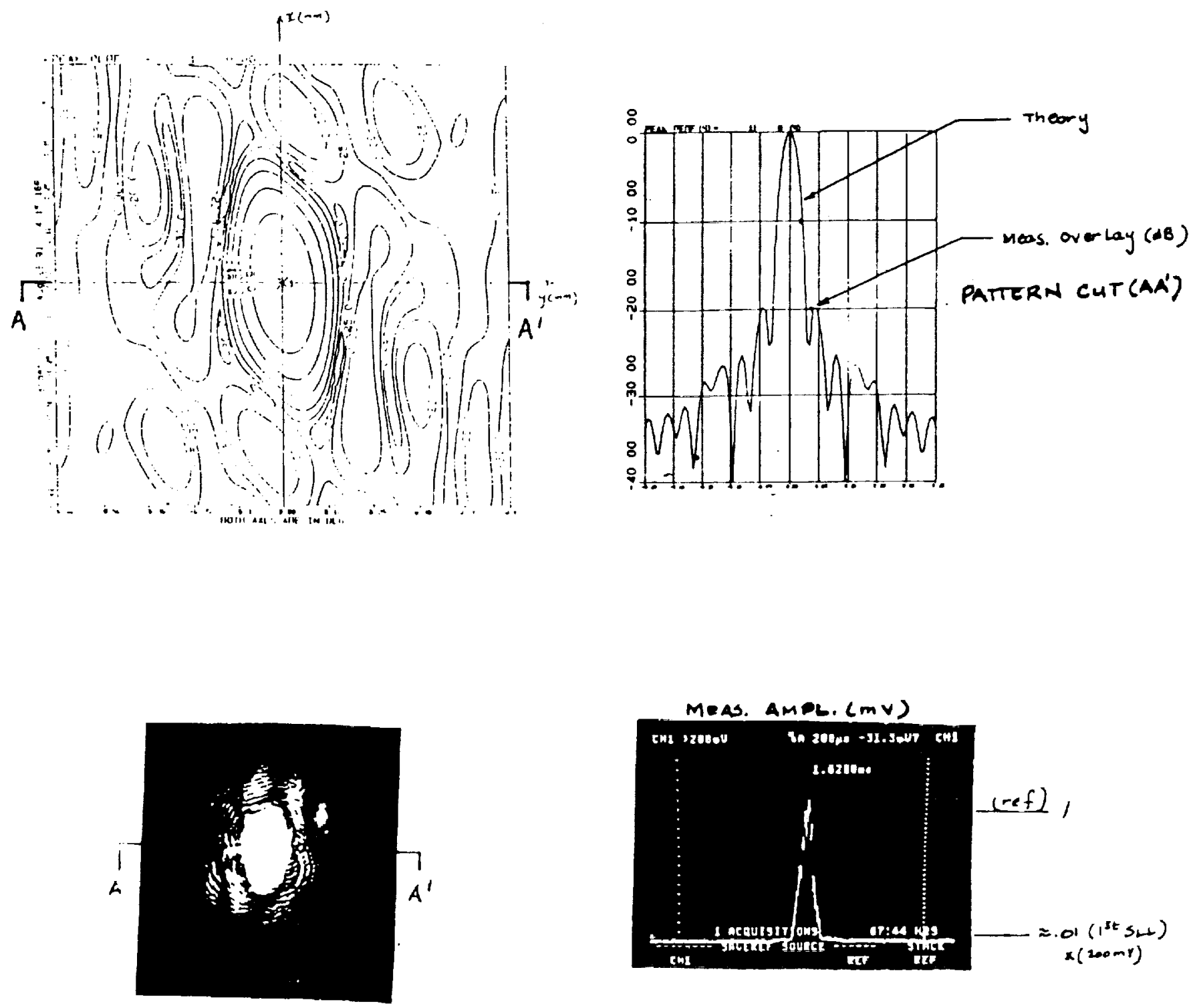

Figure 6 Computed vs. Measured CONUS aperture spectra using CCD camera and photographic film 

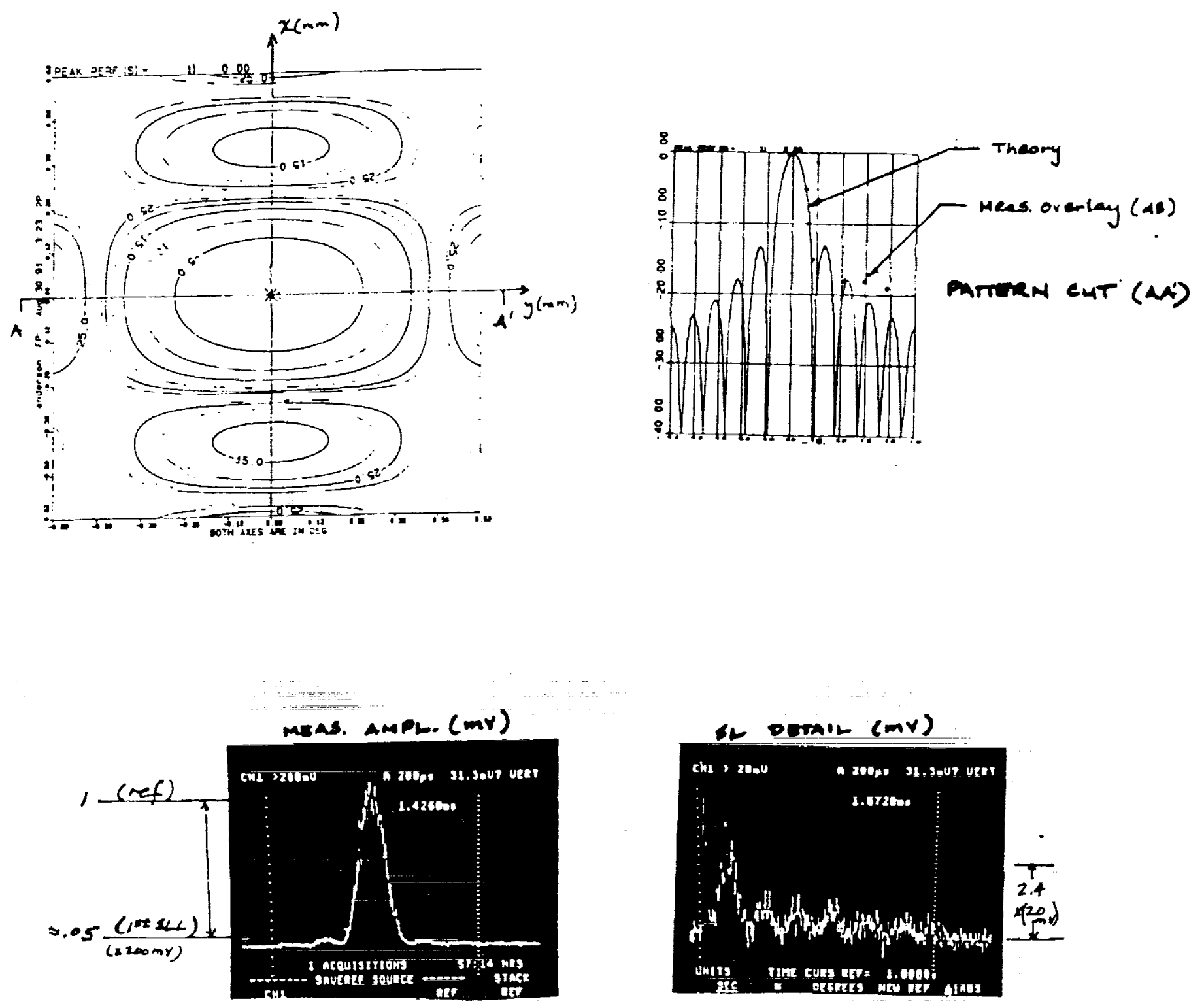

Figure 7 Computed vs. Measured Quarter CONUS aperture spectra using $\mathrm{CCD}$ camera with sidelobe detail 